PESQUIMAT, Revista de la F.C.M. de la

Universidad Nacional Mayor de San Marcos

Vol. XV N², pp. 61-69, Lima - Perú, Marzo 2013

\title{
EL ESPECTRO DE FUCIK PARA UN SISTEMA ACOPLADO CON SOLUCIONES QUE NO CAMBIAN DE SIGNO
}

\author{
Santiago César Rojas Romero ${ }^{1}$
}

Resumen: En este trabajo se estudia el espectro de Fucik para el siguiente sistema de ecuaciones diferenciales ordinarias de segundo orden

$$
\begin{cases}-u^{\prime \prime}=\lambda^{+} v^{+}-\lambda^{-} v^{-} & \text {en }\langle 0,1\rangle, \\ -v^{\prime \prime}=\lambda^{+} u^{+}-\mu^{-} u^{-} & \text {en }\langle 0,1\rangle, \\ B u=B v=0 & \text { en }\{0,1\},\end{cases}
$$

donde $B u=0$ representa las condiciones de frontera tipo Dirichlet o tipo Newmann.

Se estudia el caso en que las soluciones no triviales $(u, v)$ del problema, conservan su signo en todo el intervalo $\langle 0,1\rangle$ y se obtiene como resultado que para el problema tipo Dirichlet, el espectro de Fucik está formado por la unión de un plano y un cilindro hiperbólico, mientras que para el problema tipo Newmann el espectro está formado por los planos cartesianos.

Palabras clave: Espectro de Fucik, sistema acoplado, superficies de Fucik.

\section{THE FUCIK SPECTRUM FOR A COUPLED SYSTEM WITH NO CHANGING SIGN SOLUTIONS}

\begin{abstract}
In this work we study the Fucik spectrum for the following system of second order ordinary differential equations

$$
\begin{cases}-u^{\prime \prime}=\lambda^{+} v^{+}-\lambda^{-} v^{-} & \text {in }\langle 0,1\rangle, \\ -v^{\prime \prime}=\lambda^{+} u^{+}-\mu^{-} u^{-} & \text {in }\langle 0,1\rangle, \\ B u=B v=0 & \text { on }\{0,1\},\end{cases}
$$

where $B u=0$ represents the Dirichlet or Newmann type boundary conditions.

We study the case in which the nontrivial solutions $(u, v)$ of the problem, keep their sign in the whole interval $\langle 0,1\rangle$ and we prove: the Fucik spectrum for the Dirichlet problem is the union of a plane with an hyperbolic cylinder, while for the Newmann problem, the Fucik spectrum is formed by the cartesian planes.

Key Words: Fucik spectrum, coupled system, Fucik surfaces.
\end{abstract}

\section{Introducción}

El Espectro de Fucik para el sistema de ecuaciones diferenciales ordinarias de segundo orden

$$
\begin{cases}-u^{\prime \prime}=\lambda^{+} v^{+}-\lambda^{-} v^{-} & \text {en }\langle 0,1\rangle, \\ -v^{\prime \prime}=\lambda^{+} u^{+}-\mu^{-} u^{-} & \text {en }\langle 0,1\rangle, \\ B u=B v=0 & \text { en }\{0,1\},\end{cases}
$$

\footnotetext{
${ }^{1}$ UNMSM, Fac. de Ciencias Matemáticas, e-mail: srojasr@unmsm.edu.pe
} 
donde $B u=0$ representa las condiciones de frontera tipo Dirichlet o tipo Neumann. es el conjunto

$$
\widehat{\Sigma}=\left\{\left(\lambda^{+}, \lambda^{-}, \mu^{-}\right) \in \mathbb{R}^{3} \quad / \quad \lambda^{ \pm}, \mu^{-} \geq 0 \text { y (1) tiene soluciones no triviales }\right\} .
$$

El estudio de este espectro fue motivado por uno de los problemas formulados por Fucik [4] el cual plantea describir el conjunto de todos los pares $(a, b)$ tales que el problema de valor frontera de cuarto orden

$$
\begin{cases}u^{(i v)}=a \cdot u^{+}-b u^{-} & \text {en }\langle 0, \pi\rangle, \\ u(0)=u^{\prime \prime}(0)=u(\pi)= & u^{\prime \prime}(\pi)=0\end{cases}
$$

tiene una solución no trivial. El problema resulta equivalente a (1) con $\lambda^{+}=\mu^{-}$.

La noción de espectro de Fucik fue introducida en los trabajos de Fucik [3] y Dancer [2] para el operador $-\frac{d^{2}}{d x^{2}}$, es decir el espectro del caso escalar de (1)

$$
\begin{cases}-u^{\prime \prime}=\lambda^{+} u^{+}-\lambda^{-} u^{-} & \text {en }\langle 0,1\rangle, \\ B u==0 & \text { en }\{0,1\},\end{cases}
$$

el cual en la actualidad está completamente descrito y se conoce explícitamente sus elementos. Inclusive para condiciones de frontera más generales (ver por ejemplo Rojas [7]), se conoce el espectro de Fucik de (2).

Ahora, nuestro objetivo es estudiar el espectro de Fucik de (1) y conocer alguna parte de él.

Escribimos $\widehat{\Sigma}=\widehat{\Sigma}_{t} \cup \widehat{\Sigma}_{n t}$, donde $\widehat{\Sigma}_{t}$ denota la parte trivial

$$
\begin{aligned}
\widehat{\Sigma}_{t}=\left\{\left(\lambda^{+}, \lambda^{-}, \mu^{-}\right) \in \mathbb{R}^{3} / \lambda^{ \pm}, \mu^{-} \geq 0 \text { y }(1)\right. \text { tiene } \\
\text { soluciones no triviales que no cambian de signo }\}
\end{aligned}
$$

y $\widehat{\Sigma}_{n t}$ denota la parte no trivial

$$
\begin{aligned}
\widehat{\Sigma}_{n t}=\left\{\left(\lambda^{+}, \lambda^{-}, \mu^{-}\right) \in \mathbb{R}^{3} / \lambda^{ \pm}, \mu^{-}>0 \text { y }(1)\right. \text { tiene } \\
\text { soluciones no triviales que cambian de signo }\} .
\end{aligned}
$$

En este trabajo damos una descripción completa de $\widehat{\Sigma}_{t}$. En la sección 2, demostramos que el Espectro de Fucik $\widehat{\Sigma}$ para el sistema acoplado (1.1) es no vacío y tiene algunas propiedades de simetría. En la sección 3, a partir de (1.1) deducimos algunas identidades que utilizaremos en la demostración de nuestro resultado principal. Finalmente, en la sección 4, demostramos el resultado que permite describir $\widehat{\Sigma}_{t}$ tanto para el caso Dirichlet como para el caso Newmann.

\section{Propiedades básicas del Espectro $\widehat{\Sigma}$}

Aquí vemos que se verifican las siguientes propiedades:

Lema 2.1. El espectro de Fucik $\widehat{\Sigma}$; es no vacío.

\section{Demostración.}

Sea $\lambda_{k}$ un autovalor de $-\frac{d^{2}}{d x^{2}} \quad\left(\lambda_{k}=k^{2} \pi^{2}\right.$ para el caso Dirichlet, $\lambda_{k}=(k-1)^{2} \pi^{2}$ para el caso Newmann) y $\phi_{k}$ su respectiva autofunción. Es decir, se verifica

$$
\left\{\begin{array}{cc}
-\phi_{k}^{\prime \prime}=\lambda_{k} \phi_{k} & \text { en }\langle 0,1\rangle, \\
B \phi_{k}=0 & \text { en }\{0,1\},
\end{array}\right.
$$

donde $B=0$ representa las condiciones de frontera tipo Dirichlet o Newmann. Entonces $(u, v)=$ $\left(\phi_{k}, \phi_{k}\right)$ es solución del problema

$$
\begin{cases}-u^{\prime \prime}=\lambda_{k} v & \text { en }\langle 0,1\rangle, \\ -v^{\prime \prime}=\lambda_{k} u & \text { en }\langle 0,1\rangle, \\ B u=B v=0 & \text { en }\{0,1\} .\end{cases}
$$


Y como $\phi_{k}=\phi_{k}^{+}-\phi_{k}^{-}$, concluimos que $\left(\lambda_{k}, \lambda_{k}, \lambda_{k}\right) \in \widehat{\Sigma}, \forall k \geq 1$.

Ahora vemos que $\widehat{\Sigma}_{n t}$ tiene las siguientes simetrías.

Lema 2.2. Si $\left(\lambda^{+}, \lambda^{-}, \mu^{-}\right) \in \widehat{\Sigma}_{n t}$ con las correspondientes soluciones no triviales $(u, v)$ de (1), entonces

1. $\left(\lambda^{+}, \mu^{-}, \lambda^{-}\right) \in \widehat{\Sigma}_{n t}$ con correspondientes soluciones no triviales $(v, u)$

2. $\left(\sqrt{\lambda^{-} \mu^{-}}, \lambda^{+} \sqrt{\frac{\mu^{-}}{\lambda^{-}}}, \lambda^{+} \sqrt{\frac{\lambda^{-}}{\mu^{-}}}\right) \in \widehat{\Sigma}_{n t} \quad$ con correspondientes soluciones no triviales $\left(-u,-\sqrt{\frac{\lambda^{-}}{\mu^{-}}} v\right)$

\section{Demostración.}

1. Es inmediato de (1) .

2. Por hipótesis se tiene que

$$
\begin{array}{ll}
-u^{\prime \prime}=\lambda^{+} v^{+}-\lambda^{-} v^{-} & \text {en }\langle 0,1\rangle, \\
-v^{\prime \prime}=\lambda^{+} u^{+}-\mu^{-} u^{-} & \text {en }\langle 0,1\rangle, \\
B u=B v=0 & \text { en }\{0,1\} .
\end{array}
$$

Como $u^{+}=(-u)^{-}$y $u^{-}=(-u)^{+}$, entonces $\forall \delta>0$ se cumple

$$
\begin{array}{ll}
u^{\prime \prime}=\left(\frac{\lambda^{-}}{\delta}\right)(-\delta v)^{+}-\left(\frac{\lambda^{+}}{\delta}\right)(-\delta v)^{-} & \text {en }\langle 0,1\rangle, \\
(\delta v)^{\prime \prime}=\left(\delta \mu^{-}\right)(-u)^{+}-\left(\delta \lambda^{+}\right)(-u)^{-} & \text {en }\langle 0,1\rangle, \\
B(-u)=B(-\delta v)=0 & \text { en }\{0.1\}
\end{array}
$$

y tomando $\delta$ tal que $\frac{\lambda^{-}}{\delta}=\delta \mu^{-}$tenemos el resultado.

\section{Algunas identidades importantes}

Aquí obtenemos algunas identidades que usaremos en la siguiente sección para la construcción de $\widehat{\Sigma}_{t}$. Empezamos multiplicando la primera ecuación de (1.1) por $v$, la segunda por $u$ e integrando de 0 a 1. para obtener

$$
\int_{0}^{1} u^{\prime} v^{\prime} d t=\lambda^{+} \int_{0}^{1}\left(v^{+}\right)^{2}-\left(\lambda^{+}+\lambda^{-}\right) \int_{0}^{1}\left(v^{+}\right)\left(v^{-}\right) d t+\lambda^{-} \int_{0}^{1}\left(v^{-}\right)^{2} d t
$$

y

$$
\int_{0}^{1} u^{\prime} v^{\prime} d t=\mu^{+} \int_{0}^{1}\left(u^{+}\right)^{2}-\left(\lambda^{+}+\mu^{-}\right) \int_{0}^{1}\left(u^{+}\right)\left(u^{-}\right) d t+\mu^{-} \int_{0}^{1}\left(u^{-}\right)^{2} d t
$$

pero como $w^{+} w^{-}=0, \forall w$, entonces

$$
\begin{aligned}
\int_{0}^{1} u^{\prime} v^{\prime} d t & =\lambda^{+} \int_{0}^{1}\left(v^{+}\right)^{2}+\lambda^{-} \int_{0}^{1}\left(v^{-}\right)^{2} d t \\
& =\lambda^{+} \int_{0}^{1}\left(u^{+}\right)^{2}+\mu^{-} \int_{0}^{1}\left(u^{-}\right)^{2} d t .
\end{aligned}
$$


Ahora, multiplicando la primera ecuación de (1.1) por $u$, la segunda por $v$ e integrando de 0 a 1 . tenemos

$$
\begin{aligned}
& \int_{0}^{1}\left(u^{\prime}\right)^{2} d t=\lambda^{+} \int_{0}^{1}\left(v^{+}\right) u d t-\lambda^{-} \int_{0}^{1}\left(v^{-}\right) u d t \quad y \\
& \int_{0}^{1}\left(v^{\prime}\right)^{2} d t=\lambda^{+} \int_{0}^{1}\left(u^{+}\right) v d t-\mu^{-} \int_{0}^{1}\left(u^{-}\right) v d t
\end{aligned}
$$

Mientras que usando solo la parte positiva de $u$, obtenemos

$$
\int_{0}^{1}\left(u^{\prime}\right)\left(u^{+}\right)^{\prime} d t=\lambda^{+} \int_{0}^{1}\left(v^{+}\right)\left(u^{+}\right) d t-\lambda^{-} \int_{0}^{1}\left(v^{-}\right)\left(u^{+}\right) d t
$$

de donde

$$
\int_{0}^{1}\left|\left(u^{+}\right)^{\prime}\right|^{2} d t=\lambda^{+} \int_{0}^{1}\left(v^{+}\right)\left(u^{+}\right) d t-\lambda^{-} \int_{0}^{1}\left(v^{-}\right)\left(u^{+}\right) d t .
$$

Pero usando solo la parte negativa de $u$, obtenemos

$$
\int_{0}^{1}\left(u^{\prime}\right)\left(u^{-}\right)^{\prime} d t=\lambda^{+} \int_{0}^{1}\left(v^{+}\right)\left(u^{-}\right) d t-\lambda^{-} \int_{0}^{1}\left(v^{-}\right)\left(u^{-}\right) d t
$$

y de ahí que

$$
\int_{0}^{1}\left|\left(u^{-}\right)^{\prime}\right|^{2} d t=-\lambda^{+} \int_{0}^{1}\left(v^{+}\right)\left(u^{-}\right) d t+\lambda^{-} \int_{0}^{1}\left(v^{-}\right)\left(u^{-}\right) d t .
$$

Procediendo análogamente, con las partes positiva y negativa de $v$, obtenemos

$$
\begin{aligned}
& \int_{0}^{1}\left|\left(v^{+}\right)^{\prime}\right|^{2} d t=\lambda^{+} \int_{0}^{1}\left(u^{+}\right)\left(v^{+}\right) d t-\mu^{-} \int_{0}^{1}\left(u^{-}\right)\left(v^{+}\right) d t \quad \mathrm{y} \\
& \int_{0}^{1}\left|\left(v^{-}\right)^{\prime}\right|^{2} d t=-\lambda^{+} \int_{0}^{1}\left(u^{+}\right)\left(v^{-}\right) d t+\mu^{-} \int_{0}^{1}\left(u^{-}\right)\left(v^{-}\right) d t
\end{aligned}
$$

De otro lado, para el problema tipo Newmann, se sabe que la primera autofunción es $\phi_{1}=k \neq 0$. Multiplicando las dos ecuaciones de (1.1) por $\phi_{1}=k$ e integrando de 0 a 1 , obtenemos

$$
\begin{aligned}
0=\int_{0}^{1}\left|\phi_{1}^{\prime}\right|^{2} d t & =\lambda^{+} \int_{0}^{1}\left(v^{+}\right) \phi_{1} d t-\lambda^{-} \int_{0}^{1}\left(v^{-}\right) \phi_{1} d t \quad \text { y } \\
& =\lambda^{+} \int_{0}^{1}\left(u^{+}\right) \phi_{1} d t-\mu^{-} \int_{0}^{1}\left(u^{-}\right) \phi_{1} d t
\end{aligned}
$$

de donde

$$
\lambda^{+} \int_{0}^{1} v^{+} d t=\lambda^{-} \int_{0}^{1} v^{-} d t \quad \text { y } \quad \lambda^{+} \int_{0}^{1} u^{+} d t=\mu^{-} \int_{0}^{1} u^{-} d t .
$$

Sin embargo, para el problema tipo Dirichlet no llegamos a ecuaciones análogas. Más bien, obtenemos otras identidades también muy útiles. Multiplicando ambas ecuaciones en (1.1) por $\phi_{1}$ e integrando de 0 a 1 , obtenemos

$$
\begin{aligned}
& \lambda^{+} \int_{0}^{1} v^{+} \phi_{1} d t-\lambda^{-} \int_{0}^{1} v^{-} \phi_{1} d t=\int_{0}^{1} u^{\prime} \phi_{1}^{\prime} d t=-\int_{0}^{1} u \phi_{1}^{\prime \prime} d t=\lambda_{1} \int_{0}^{1}\left(u^{+}-u^{-}\right) \phi_{1} d t \\
& \lambda^{+} \int_{0}^{1} u^{+} \phi_{1} d t-\mu^{-} \int_{0}^{1} u^{-} \phi_{1} d t=\int_{0}^{1} v^{\prime} \phi_{1}^{\prime} d t=-\int_{0}^{1} v \phi_{1}^{\prime \prime} d t=\lambda_{1} \int_{0}^{1}\left(v^{+}-v^{-}\right) \phi_{1} d t
\end{aligned}
$$


Sumando y luego restando estas dos últimas ecuaciones, llegamos a

$$
\begin{aligned}
\left(\lambda^{+}-\lambda_{1}\right) \int_{0}^{1} v^{+} \phi_{1} d t+\left(\lambda^{+}-\lambda_{1}\right) \int_{0}^{1} u^{+} \phi_{1} d t & \\
& =\left(\lambda^{-}-\lambda_{1}\right) \int_{0}^{1} v^{-} \phi_{1} d t+\left(\mu^{-}-\lambda_{1}\right) \int_{0}^{1} u^{-} \phi_{1} d t
\end{aligned}
$$

y

$$
\begin{aligned}
\left(\lambda^{+}+\lambda_{1}\right) \int_{0}^{1} v^{+} \phi_{1} d t-\left(\lambda^{+}+\lambda_{1}\right) \int_{0}^{1} u^{+} \phi_{1} d t & \\
& =\left(\lambda^{-}+\lambda_{1}\right) \int_{0}^{1} v^{-} \phi_{1} d t-\left(\mu^{-}+\lambda_{1}\right) \int_{0}^{1} u^{-} \phi_{1} d t
\end{aligned}
$$

\section{Soluciones que no cambian de signo}

Aquí se deducen las propiedades de las soluciones no triviales de (1.1) correspondientes a los puntos en $\widehat{\Sigma}_{t}$. Primero consideramos el caso Dirichlet.

Proposición 4.1. Sea $(u, v)$ una solución de (1) con condiciones de frontera tipo Dirichlet y coeficientes $\lambda^{ \pm} y \mu^{-}$, entonces

1. Ambas $u$ y $v$ cambian de signo o ninguna de ellas.

2. Si $u$ y $v$ no cambian de signo, entonces ambas son múltiplos no nulos de $\phi_{1}$ y uno de los coeficientes es igual a $\lambda_{1}$ mientras que los otros dos pueden ser cualquier número real. En particular, si normalizamos las autofunciones, tenemos los casos

$$
\begin{gathered}
u=v=\phi_{1} \quad y \quad \lambda^{+}=\lambda_{1}, \\
u=v=-\phi_{1} \quad y \quad \lambda^{-}=\mu^{-}=\lambda_{1},
\end{gathered}
$$

\section{Demostración.}

1. Supongamos que $u$ no cambia de signo. Sin pérdida de generalidad, supongamos que $u \geq 0$ y $\lambda^{+} \geq 0$. De (11) tenemos que $\int_{0}^{1}\left|\left(v^{-}\right)^{\prime}\right|^{2} \leq 0$. De ahí que $\left(v^{-}\right)^{\prime}=0$, luego $v^{-}=k$ (constante). De esto se deduce que $v^{-}=0$ y por tanto $v \geq 0$ en $[0,1]$. Entonces $v$ no cambia de signo. Análogamente se demuestra que si $u<0$ también $v<0$ en $[0,1]$.

Ahora supongamos que $u$ cambia de signo, entonces $u^{+} \neq 0$ y $u^{-} \neq 0$.

Si $v \geq 0$, entonces $v^{-}=0$ y de (8) y (9) tendríamos $\lambda^{+}=0, u^{+}=k_{1}$ y $u^{-}=k_{2}$, con lo cual $u=k$ (constante), lo cual es una contradicción pues $u^{+} \neq 0$. Lo mismo ocurre si $v \leq 0$. Por tanto, $v$ cambia de signo.

2. Sean $u$ y $v$ que no cambian de signo. Por la parte 1 , solo debemos considerar los casos en que $u$ y $v$ tienen el mismo signo.

- Caso $u, v \geq 0$ :

Por (6), tenemos que $\lambda^{+}>0$.

Afirmación: $\lambda^{+}=\lambda_{1}$.

En efecto, de (13) tenemos

$$
\left(\lambda^{+}-\lambda_{1}\right) \underbrace{\left[\int_{0}^{1} v \phi_{1} d t+\int_{0}^{1} u \phi_{1} d t\right]}_{>0}=0,
$$


y de ahí que $\lambda^{+}=\lambda_{1}$. Luego, el sistema (1) queda como

$$
\begin{cases}-u^{\prime \prime}=\lambda_{1} v & \text { en }\langle 0,1\rangle, \\ -v^{\prime \prime}=\lambda_{1} u & \text { en }\langle 0,1\rangle, \\ u(0)=u(1)=0 & , \quad v(0)=v(1)=0,\end{cases}
$$

el cual es equivalente a

$$
\begin{cases}u^{(i v)}=\lambda_{1}^{2} u & \text { en }\langle 0,1\rangle, \\ v^{(i v)}=\lambda_{1}^{2} v & \text { en }\langle 0,1\rangle, \\ u(0)=u(1)=u^{\prime \prime}(0)=u^{\prime \prime}(1)=0, \\ v(0)=v(1)=v^{\prime \prime}(0)=v^{\prime \prime}(1)=0,\end{cases}
$$

cuya solución general es

$$
\begin{gathered}
u(t)=A e^{\sqrt{\lambda_{1}} t}+B e^{-\sqrt{\lambda_{1}} t}+C \cos \left(\sqrt{\lambda_{1}} t\right)+D \operatorname{sen}\left(\sqrt{\lambda_{1}} t\right), \\
v(t)=-A e^{\sqrt{\lambda_{1}} t}-B e^{-\sqrt{\lambda_{1}} t}+C \cos \left(\sqrt{\lambda_{1}} t\right)+D \operatorname{sen}\left(\sqrt{\lambda_{1}} t\right) .
\end{gathered}
$$

De ahí por las condiciones de frontera resulta

$$
u(t)=v(t)=D \operatorname{sen}\left(\sqrt{\lambda_{1}} t\right),
$$

es decir $u$ y $v$ son múltiplos de $\phi_{1}$. Más aún, normalizando, tenemos que $\lambda^{+}=\lambda_{1} \quad \mathrm{y}$ $u=v=\phi_{1}$.

- Caso $u, v<0$ :

Por (6) y (7), tenemos que $\lambda^{-}>0$ y $\mu^{-}>0$.

Afirmación: $\lambda^{-}=\delta \lambda_{1}$ y $\mu^{-}=\frac{1}{\delta} \lambda_{1}$, para algún $\delta>0$.

En efecto, como $u=-A_{1} \phi_{1}, v=-A_{2} \phi_{1}, A_{1}, A_{2}>0$, de (13) y (14) tenemos

$$
\begin{aligned}
& A_{1}\left(\lambda^{-}-\lambda_{1}\right)+A_{2}\left(\mu^{-}-\lambda_{1}\right)=0, \\
& A_{1}\left(\lambda^{-}+\lambda_{1}\right)-A_{2}\left(\mu^{-}+\lambda_{1}\right)=0 .
\end{aligned}
$$

De ahí, sumando y restando estas dos ecuaciones, llegamos a

$$
\lambda^{-}=\frac{A_{2}}{A_{1}} \lambda_{1} \quad \text { y } \quad \mu^{-}=\frac{A_{1}}{A_{2}} \lambda_{1}
$$

con lo cual se tiene la afirmación.

Así resulta $\lambda^{-} \mu^{-}=\lambda_{1}^{2}, \delta=\sqrt{\frac{\lambda^{-}}{\mu^{-}}}, y$ el sistema (1) queda como

$$
\begin{cases}-u^{\prime \prime}=\delta \lambda_{1} v & \text { en }\langle 0,1\rangle, \\ -v^{\prime \prime}=\frac{\lambda_{1}}{\delta} u & \text { en }\langle 0,1\rangle, \\ u(0)=u(1)=0 & , \quad v(0)=v(1)=0 .\end{cases}
$$

Resolviendo como se hizo en el item anterior, obtenemos

$$
u=-k \phi_{1} \quad \text { y } \quad v=-\frac{k}{\delta} \phi_{1} \quad, k>0 .
$$

Luego $u=\delta v$, es decir $u=\sqrt{\frac{\lambda^{-}}{\mu^{-}}} v=-k \phi_{1}, k>0 . \mathrm{Y}$ normalizando, concluimos que $\lambda^{-}=\mu^{-}=\lambda_{1} \quad$ y $\quad u=v=-\phi_{1}$. 
Ahora veremos el resultado correspondiente al caso Newmann.

Proposición 4.2. Sea $(u, v)$ una solución de (1) con condiciones de frontera tipo Neumann y coeficientes $\lambda^{ \pm} y \mu^{-}$, entonces

1. Ambas u y $v$ cambian de signo o ninguna de ellas.

2. Si u y $v$ no cambian de signo, entonces ambas son múltiplos de $\phi_{1}$ (una de ellas puede ser cero) y uno de los coeficientes es $\lambda_{1}=0$, mientras que los otros pueden ser cualquier número real. Si ambas $u$ y $v$ no son cero, entonces dos de los coeficientes deben ser $\lambda_{1}=0$. En particular, si normalizamos las autofunciones tenemos los siguientes casos

$$
\begin{array}{rll}
u=v=\phi_{1} & y & \lambda^{+}=0, \\
u=v=-\phi_{1} & y & \lambda^{-}=\mu^{-}=0, \\
u=\phi_{1}\left(\text { resp. } u=-\phi_{1}\right), v=0 & y & \lambda^{+}=0\left(\text { resp. } \mu^{-}=0\right), \\
u=0, v=\phi_{1}\left(\text { resp. } v=-\phi_{1}\right) & y & \lambda^{+}=0\left(\text { resp. } \lambda^{-}=0\right) .
\end{array}
$$

Demostración. Aquí (12) es de gran utilidad, en particular porque de ahí se deduce que $\lambda^{+}, \lambda^{-}$y $\mu^{-}$tienen el mismo signo.

1. Sea $u$ que no cambia de signo. Supongamos $u \geq 0$, esto es $u^{+}=u$ y $u^{-}=0$. De (12) tenemos que $\lambda^{+}=0$. Luego, la segunda ecuación de (1) queda $v^{\prime \prime}=0$ y por las condiciones de frontera resulta que $v$ es constante, es decir $v$ no cambia de signo.

2. Sean $u$ y $v$ que no cambian de signo. Consideremos el caso $u, v \geq 0$, esto es $u^{+}=u, u^{-}=0, v^{+}=v, v^{-}=0$. De (12) resulta $\lambda^{+}=\mu^{+}=0$, con lo cual el sistema (1) queda como

$$
\left\{\begin{aligned}
u^{\prime \prime} & =0 \quad \text { en }\langle 0,1\rangle, \\
v^{\prime \prime} & =0 \quad \text { en }\langle 0,1\rangle, \\
u^{\prime}(0) & =u^{\prime}(1)=0, v^{\prime}(0)=v^{\prime}(1)=0
\end{aligned}\right.
$$

y de ahí que $u$ y $v$ son constantes. Luego, $u=k_{1} \phi_{1} \quad$ y $\quad v=k_{2} \phi_{1} \quad\left(\phi_{1}=k\right.$ para el caso Neumann). En particular $u=\phi_{1} \quad \mathrm{y} \quad v=\phi_{1}$.

El caso $u=0$ nos da $\lambda^{+}=0$ y $v=k_{2} \phi_{1}$. En particular, cuando $\lambda^{+}=0$ tenemos $u=0$ y $v=\phi_{1}$. Los demás casos se prueban análogamente.

Así, hemos demostrado que la parte trivial $\widehat{\Sigma}_{t}$ tiene la siguiente forma:

- Para el caso Dirichlet

$$
\widehat{\Sigma}_{t}=\left\{\lambda^{+}=\lambda_{1}\right\} \cup\left\{\lambda^{-}, \mu^{-}>0, \lambda^{-} \mu^{-}=\lambda_{1}^{2}\right\}
$$

donde el plano $\left\{\lambda^{+}=\lambda_{1}\right\}$ corresponde a la familia de soluciones $u=v=k \phi_{1}, k>0$, mientras que la superficie $\left\{\lambda^{-}, \mu^{-}>0, \lambda^{-} \mu^{-}=\lambda_{1}^{2}\right\}$ corresponde a la familia $u=\sqrt{\frac{\lambda^{-}}{\mu^{-}}} v=$ $-k \phi_{1}, k>0$.

- Para el caso Neumann

$$
\widehat{\Sigma}_{t}=\left\{\lambda^{+}=0\right\} \cup\left\{\lambda^{-}=0\right\} \cup\left\{\mu^{-}=0\right\}
$$

donde el plano $\left\{\lambda^{+}=0\right\}$ corresponde a las soluciones $\left\{u=k_{1} \phi_{1}, v=k_{2} \phi_{1}, k_{1}, k_{2} \geq 0\right\}$, el plano $\left\{\lambda^{-}=0\right\}$ corresponde a las soluciones $\left\{u=0, v=-\phi_{1}\right\}$ y el plano $\left\{\mu^{-}=0\right\}$ corresponde a las soluciones $\left\{u=-\phi_{1}, v=0\right\}$. 


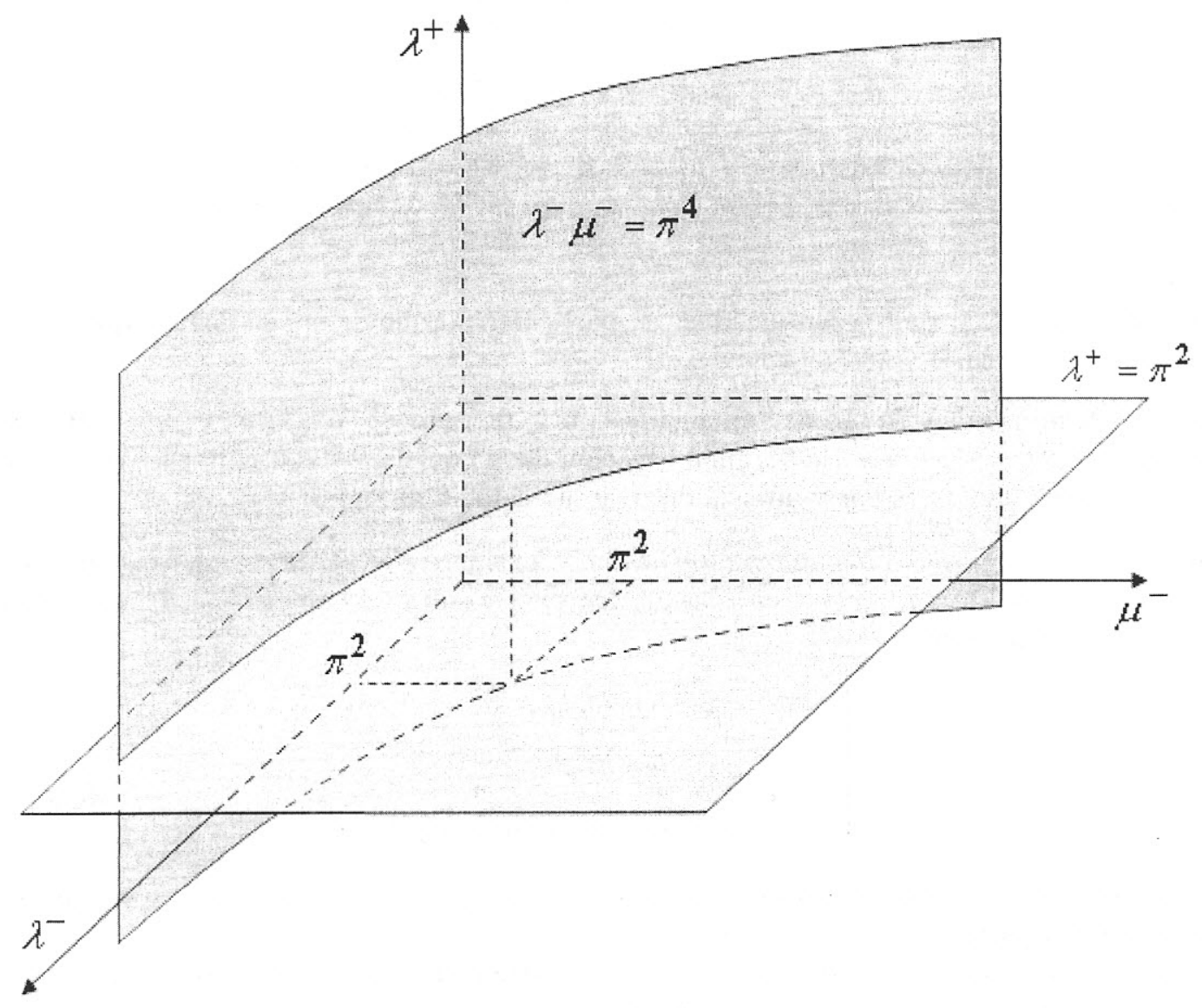

Espectro de Fucik $\widehat{\Sigma}_{t}$ : Caso Dirichlet 


\section{REFERENCIAS BIBLIOGRÁFICAS}

[1] Campos J. y Dancer E. N. On the resonance set in a fourth-order equation with jumping nonlinearity. Differential Integral Equations 14(3) (2001), 257-272.

|2| Dancer E. N. On the Dirichlet problem for weakly nonlinear elliptic partial differental equations. Proc. Royal Soc. Edinburgh Sect A(4) (1976/1977), 283-300.

|3| Fucik S. Boundary value problem with jumping nonlinearities. Casopis Pest. Mat 101 (1) (1976). 69-87.

[4] Fucik S. Solvability of Nonlinear Equations and Boundary Value Problems. D. Reidel Publishing Company, Dordrecht, 1980.

[5] Massa E. On a variational characterization of the Fucik Spectrum of the Laplacian and a superlinear Sturm-Liouville equation. Proc. Roy. Soc. Edinburgh Sect A 134(3) (2004), $557-577$.

[6] Massa E. On the Fucik Spectrum and superlinear elliptic equations. PhD. Thesis, Universitá degli Studi di Milano, Italy) (2003).

[7] Rojas S. Espectro de Fucik para el problema de valor frontera Sturm-Liouville. Pesquimat, revista de investigación de la Fac. Ciencias Mat. UNMSM Vol IX, no. 2 , 31-49, 2006.

[8] Ruf B. On nonlinear elliptic problems with jumping nonliearities. Ann. Mat. Pure Appl. (4) 128 (1981) $133-151$.

[9] Rynne. P. The Fucik Spectrum of general Sturm-Liouville problems. Journal of Differential Equations 161 (2000), 87-109.

[10] Schechter M. The Fucik Spectrum. Indiana University Mathematics Joumal, Vol. 43 No. 4 (1994), 1139-1157. 